\title{
Enterovirus 71 encephalomyelitis and Japanese encephalitis can be distinguished by topographic distribution of inflammation and specific intraneuronal detection of viral antigen and RNA
}

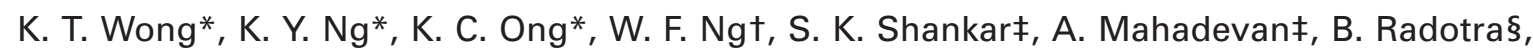

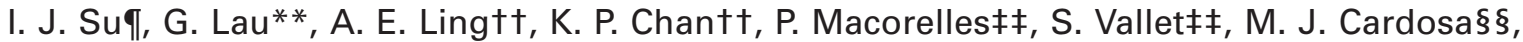

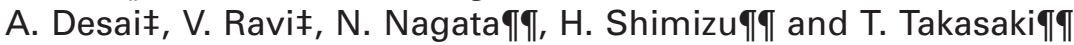

*Faculty of Medicine, University of Malaya, Kuala Lumpur, and §§Universiti Sarawak Malaysia, Kuching, Malaysia, †Yan Chai Hospital, Hong Kong, China, \$NIMHANS, Bangalore, and \$PGIMER, Chandigarh, India, qNational Cheng Kung University, Taichung, Taiwan, ${ }^{* *}$ Centre for Forensic Science, and $+\dagger$ Singapore General Hospital, Singapore, t+Centre Hospitalier Universitaire, Brest, France, and $\mathbf{9} \mid$ National Institute of Infectious Diseases, Tokyo, Japan

K. T. Wong, K. Y. Ng, K. C. Ong, W. F. Ng, S. K. Shankar, A. Mahadevan, B. Radotra, I. J. Su, G. Lau, A. E. Ling, K. P. Chan, P. Macorelles, S. Vallet, M. J. Cardosa, A. Desai, V. Ravi, N. Nagata, H. Shimizu and T. Takasaki (2012) Neuropathology and Applied Neurobiology 38, 443-453

Enterovirus 71 encephalomyelitis and Japanese encephalitis can be distinguished by topographic distribution of inflammation and specific intraneuronal detection of viral antigen and RNA

Aims: To investigate if two important epidemic viral encephalitis in children, Enterovirus 71 (EV71) encephalomyelitis and Japanese encephalitis (JE) whose clinical and pathological features may be nonspecific and overlapping, could be distinguished. Methods: Tissue sections from the central nervous system of infected cases were examined by light microscopy, immunohistochemistry and in situ hybridization. Results: All 13 cases of EV71 encephalomyelitis collected from Asia and France invariably showed stereotyped distribution of inflammation in the spinal cord, brainstem, hypothalamus, cerebellar dentate nucleus and, to a lesser extent, cerebral cortex and meninges. Anterior pons, corpus striatum, thalamus, temporal lobe, hippocampus and cerebellar cortex were always uninflamed. In contrast, the eight JE cases studied showed inflammation involving most neuronal areas of the central nervous system, including the areas that were uninflamed in EV71 encephalomyelitis. Lesions in both infections were nonspecific, consisting of perivascular and parenchymal infiltration by inflammatory cells, oedematous/necrolytic areas, microglial nodules and neuronophagia. Viral inclusions were absent. Conclusions: Immunohistochemistry and in situ hybridization assays were useful to identify the causative virus, localizing viral antigens and RNA, respectively, almost exclusively to neurones. The stereotyped distribution of inflammatory lesions in EV71 encephalomyelitis appears to be very useful to help distinguish it from JE.

Keywords: diagnosis, Enterovirus 71 encephalomyelitis, Japanese encephalitis virus, pathology

Correspondence: Kum Thong Wong, Department of Pathology, Faculty of Medicine, University of Malaya, 50603 Kuala Lumpur, Malaysia. Tel: +60 37949 2064; Fax: +60 37955 6845; E-mail: wongkt@ummc.edu.my

\section{Introduction}

Despite advances in general medicine and vaccine development, frequent outbreaks of epidemic viral encephalitides continue to be reported globally [1-5]. In the paediatric age group, apart from HIV encephalitis, 